ESAIM: COCV 27 (2021) 29

https://doi.org/10.1051/cocv/2021005
ESAIM: Control, Optimisation and Calculus of Variations

www.esaim-cocv.org

\title{
LOCALIZATION OF ENERGY AND LOCALIZED CONTROLLABILITY*
}

\author{
F.W. Chaves-Silva ${ }^{1}$, J.-P. Puel ${ }^{2, * *}$ and M.C. Santos ${ }^{3}$
}

\begin{abstract}
We will consider both the controlled Schrödinger equation and the controlled wave equation on a bounded open set $\Omega$ of $\mathbb{R}^{N}$ during an interval of time $(0, T)$, with $T>0$. The control is distributed and acts on a nonempty open subdomain $\omega$ of $\Omega$. On the other hand, we consider another open subdomain $D$ of $\Omega$ and the localized energy of the solution in $D$. The first question we want to study is the possibility of obtaining a prescribed value of this local energy at time $T$ by choosing the control adequately. It turns out that this question is equivalent to a problem of exact or approximate controllability in $D$, which we call localized controllability and which is the second question studied in this article. We obtain sharp results on these two questions and, of course, the answers will require conditions on $\omega$ and $T$ which will be given precisely later on.
\end{abstract}

Mathematics Subject Classification. 93B05, 93B07, 35K40, 35L51.

Received January 29, 2020. Accepted January 6, 2021.

\section{INTRODUCTION}

Exact controllability (in the whole domain) for the wave equation and for Schrödinger equation has been extensively studied in the last thirty years, in particular after the introduction by Lions in [11] of the so-called Hilbert Uniqueness Method which is based on the obtention of an observability inequality for the adjoint equation. Several geometric conditions were introduced successively in order to satisfy this observability inequality. In [1], Bardos, Lebeau and Rauch introduced a celebrated sufficient and almost necessary Geometric Control Condition (GCC) on the domain of study $\Omega$ and the time $T$ which was proved to be necessary and sufficient by Burq and Gerard in [3]. For Schrödinger equation, the first results were given by Machtyngier in [12] and Lebeau in [7].

Here we are interested in a problem of controllability only in a subdomain and we look for weaker geometric conditions to ensure exact or approximate localized controllability. We also remark that this question is equivalent to a problem of prescribed localized energy for the solution of our equations. In fact, the motivation for this present study was this question of prescribed localized energy, in particular for the Schrödinger equation

${ }^{*}$ F.W. Chaves-Silva and M.C. Santos were partially supported by CNPq-Brazil, by the Grant 2019/0014 of Paraba State Research Foundation (FAPESQ-PB) and by the program CAPES/MATH-AMSUD under the project ACIPDE number: 8881.368715/2019-01.

Keywords and phrases: Wave equation, Schrödinger equation, localized energy, localized controllability.

1 Mathematics Department, Universidade Federal da Paraíba, João Pessoa, PB 58051-900, Brazil.

2 Laboratoire de Mathématiques de Versailles, Université de Versailles St Quentin, 45 avenue des Etats Unis, 78035 Versailles, France.

3 Mathematics Department, Universidade Federal da Paraíba, João Pessoa, PB 58051-900, Brazil.

** Corresponding author: jppuel@math.uvsq.fr

(C) The authors. Published by EDP Sciences, SMAI 2021

This is an Open Access article distributed under the terms of the Creative Commons Attribution License (https://creativecommons.org/licenses/by/4.0), which permits unrestricted use, distribution, and reproduction in any medium, provided the original work is properly cited. 
as it seems important, for example in quantum chemistry, to focus the energy of the solution in a prescribed region.

The controls we consider will be acting on a subset $\omega \times(0, T)$ of the physical domain. We will use the notation $L^{2}\left(0, T ; L^{2}(\omega)\right)$ to recall this condition of support but of course, functions in this space are understood to be extended by 0 outside $\omega \times(0, T)$.

To be more precise, we consider $T>0$, a bounded open set $\Omega$ of $\mathbb{R}^{N}(N \geq 1)$ with boundary $\Gamma$ and two open subsets of $\Omega$ called $\omega$ and $D$. In a first step we will study the following controlled Schrödinger equation with distributed control acting in $\omega$ :

$$
\mid \begin{array}{ll}
i \frac{\partial y}{\partial t}+\Delta y=v \mathbb{I}_{\omega} & \text { in } \Omega \times(0, T), \\
y=0 & \text { on } \Gamma \times(0, T), \\
y(0)=y_{0} & \text { in } \Omega,
\end{array}
$$

where $y_{0} \in L^{2}(\Omega)$ and $\mathbb{I}_{\omega}$ denotes the characteristic function of $\omega$. We know that for $v \in L^{1}\left(0, T ; L^{2}(\omega)\right)$ the solution $y$ of (1.1) satisfies

$$
y \in C\left([0, T] ; L^{2}(\Omega)\right)
$$

We define the localized energy in $D$ at time $t$ as

$$
E_{D}^{S}(y)(t)=\int_{D}|y(t, x)|^{2} \mathrm{~d} x
$$

The first problem we will study is the following.

Problem 1.1. Given $\alpha \geq 0$, can we find $v \in L^{2}\left(0, T ; L^{2}(\omega)\right)$ such that

$$
E_{D}^{S}(y)(T)=\alpha ?
$$

For $\alpha>0$ we will give a positive answer to this question. For $\alpha=0$, up to our knowledge, the problem remains open.

This will be related to the following problem of localized controllability which will be the second problem studied in this article.

Problem 1.2. Given $\alpha>0$, can we find $v \in L^{2}\left(0, T ; L^{2}(\omega)\right)$ such that

$$
E_{D}^{S}(y)(T)=|y(T)|_{L^{2}(D)}^{2} \leq \alpha ?
$$

Of course, Problem 1.2 is included in Problem 1.1 but Problem 1.1 is more precise.

We will give a positive answer for this problem of approximate localized controllability for $T>0$ and any nonempty open subset $\omega$ (and no condition on $D$ ). 
In a second step we will study the analogous problems for the controlled wave equation. For this purpose, we will assume $\Omega$ to be of class $C^{\infty}$.

$$
\mid \begin{array}{ll}
\frac{\partial^{2} y}{\partial t^{2}}-\Delta y=v \mathbb{I}_{\omega} & \text { in } \Omega \times(0, T) \\
y=0 & \text { on } \Gamma \times(0, T) \\
y(0)=y_{0} ; & \frac{\partial y}{\partial t}(0)=y_{1}
\end{array}
$$

We know that for $y_{0} \in H_{0}^{1}(\Omega), y_{1} \in L^{2}(\Omega)$ and $v \in L^{1}\left(0, T ; L^{2}(\omega)\right)$ the solution $y$ of (1.4) satisfies

$$
y \in C\left([0, T] ; H_{0}^{1}(\Omega)\right) \cap C^{1}\left([0, T] ; L^{2}(\Omega)\right) .
$$

We define the localized energy in $D$ at time $t$ by

$$
E_{D}^{W}(y)(t)=\frac{1}{2} \int_{D}\left(\left|\frac{\partial y}{\partial t}(t, x)\right|^{2}+|\nabla y(t, x)|^{2}\right) \mathrm{d} x
$$

The first question of localized energy for the wave equation is

Problem 1.3. Given $\alpha \geq 0$, can we find $v \in L^{2}\left(0, T ; L^{2}(\omega)\right)$ such that

$$
E_{D}^{W}(y)(T)=\alpha ?
$$

The second question of (exact) localized controllability is

Problem 1.4. Can we find $v \in L^{2}\left(0, T ; L^{2}(\omega)\right)$ such that

$$
y(T)=0 \text { and } \frac{\partial y}{\partial t}(T)=0 \text { in } D ?
$$

Again here Problem 1.4 is a special case of Problem 1.3 but Problem 1.3 seems more general.

These two questions will turn out to be equivalent and we will give a sufficient geometric condition on $D, \omega$ and $T$ in order to obtain a positive answer to these problems.

Concerning Problem 1.4, we recently heard about a very similar work by He [5] on the localized controllability of the wave equation. He gives a geometric condition which is quite similar to ours (but still somewhat different), his strategy of proof is classical and similar to ours but the second part of the proof, namely the strategy leading to the compactness-uniqueness argument is given without any detail in his article.

This article is structured as follows.

In a first section we treat the case of the Schrödinger equation. We give the main result for the case of the Schrödinger equation and we show the relation between the prescribed localized energy and the localized controllability. We then prove the result concerning localized controllability for the Schrödinger equation.

In Section 3 we consider the case of the wave equation. We state the localized geometric control condition and give the main result concerning the wave equation. Again here we show the relation between the prescribed localized energy and exact localized controllability. We then give the complete proof of exact localized controllability for the wave equation.

We have considered here problems with distributed controls in order to give a classical sense to the energies. We can obtain analogous results for the case of boundary controls but then we have to change the notions of energies. 


\section{CASE OF THE Schrödinger EQUATION}

Throughout this section, all functions have complex values.

\subsection{Main result and relation between localized energy and localized controllability}

We will prove the following result.

Theorem 2.1. Let $\omega$ and $D$ be any two non empty open subsets of $\Omega$ and let $T>0$ be given. Then for any $y_{0} \in L^{2}(\Omega)$ and any $\alpha>0$, there exists a control $v \in L^{2}\left(0, T ; L^{2}(\omega)\right)$ such that

$$
E_{D}^{S}(y(v))(T)=\alpha
$$

Moreover, for any $\alpha^{\prime} \geq \alpha$, with the same control $v$, we can choose $\lambda \in \mathbb{C}$ such that

$$
E_{D}^{S}(y(\lambda v))(T)=\alpha^{\prime}
$$

Remark 2.2. To our knowledge, the case $\alpha=0$ is an open problem.

Let us split the solution $y$ of (1.1) in two parts

$$
y=y(v)=z+w(v)
$$

where $z$ and $w(v)$ are solutions of

$$
\mid \begin{array}{ll}
i \frac{\partial z}{\partial t}+\Delta z=0 & \text { in } \Omega \times(0, T) \\
z=0 & \text { on } \Gamma \times(0, T), \\
z(0)=y_{0} & \text { in } \Omega,
\end{array}
$$

and

$$
\mid \begin{array}{ll}
i \frac{\partial w(v)}{\partial t}+\Delta w(v)=v \mathbb{I}_{\omega} & \text { in } \Omega \times(0, T) \\
w(v)=0 & \text { on } \Gamma \times(0, T), \\
w(v)(0)=0 & \text { in } \Omega,
\end{array}
$$

respectively.

We know that

$$
\int_{D}|z(T, x)|^{2} \mathrm{~d} x \leq \int_{\Omega}|z(T, x)|^{2} \mathrm{~d} x=\int_{\Omega}\left|y_{0}(x)\right|^{2} \mathrm{~d} x
$$

and

$$
\forall \lambda \in \mathbb{C}, w(\lambda v)=\lambda w(v) .
$$

Define

$$
y_{\lambda}(v)=y(\lambda v)=z+\lambda w(v) .
$$


We have

$$
\int_{D}\left|y_{\lambda}(v)(T, x)\right|^{2} \mathrm{~d} x=\int_{D}|z(T, x)|^{2} \mathrm{~d} x+2 \Re\left(\bar{\lambda} \int_{D} z(T, x) \bar{w}(v)(T, x) \mathrm{d} x\right)+|\lambda|^{2} \int_{D}|w(v)(T, x)|^{2} \mathrm{~d} x .
$$

If we choose

$$
\lambda=\lambda(v)=\mu e^{i \theta(v)}, \mu \in \mathbb{R}, \theta(v) \in \mathbb{R}
$$

such that

$$
\bar{\lambda}(v) \int_{D} z(T, x) \bar{w}(v)(T, x) \mathrm{d} x=-\mu\left|\int_{D} z(T, x) \bar{w}(v)(T, x) \mathrm{d} x\right|,
$$

we obtain

$$
\int_{D}\left|y_{\lambda}(v)(T, x)\right|^{2} \mathrm{~d} x=\int_{D}|z(T, x)|^{2} \mathrm{~d} x-2 \mu\left|\int_{D} z(T, x) \bar{w}(v)(T, x) \mathrm{d} x\right|+|\mu|^{2} \int_{D}|w(v)(T, x)|^{2} \mathrm{~d} x
$$

We will see later on (see Rem. 2.4) that we can choose $v$ such that $\int_{D}|w(v)(T, x)|^{2} \mathrm{~d} x \neq 0$ and we will restrict ourselves to such functions $v$.

Then, when $\mu=|\lambda| \rightarrow+\infty$, we have that $\int_{D}\left|y_{\lambda}(v)(T, x)\right|^{2} \mathrm{~d} x \rightarrow+\infty$.

We now have to find the minimum of $\int_{D}\left|y_{\lambda}(v)(T, x)\right|^{2} \mathrm{~d} x$ which is achieved for

$$
\mu=\mu(v)=\frac{\left|\int_{D} z(T, x) \bar{w}(v)(T, x) \mathrm{d} x\right|}{\int_{D}|w(v)(T, x)|^{2} \mathrm{~d} x} .
$$

The value obtained is

$$
\alpha(v)=\int_{D}|z(T, x)|^{2} \mathrm{~d} x-\frac{\left|\int_{D} z(T, x) \bar{w}(v)(T, x) \mathrm{d} x\right|^{2}}{\int_{D}|w(v)(T, x)|^{2} \mathrm{~d} x} .
$$

The question now is: can we find $v \in L^{2}\left(0, T ; L^{2}(\omega)\right)$ such that

$$
\alpha(v) \leq \alpha ?
$$

Of course the case $\alpha=0$ corresponds to find $v \in L^{2}\left(0, T ; L^{2}(\omega)\right)$ such that $y(T, x)=0$ in $D$ and it is a problem of localized exact controllability for the Schrödinger equation. This question should require some geometric condition on $D$ and $\omega$ and it seems to be an open problem up to now. It will be solved in section 3 for the wave equation and we will give there some comments about the arguments which are valid for the wave equation and not for the Schrödinger equation.

The case $\alpha>0$ will be solved via an approximate controllability result in the next section.

\subsection{Approximate controllability for the Schrödinger equation and proof of Theorem 2.1}

We give here an approximate controllability result for the Schrödinger equation. This result uses a very classical argument and should be well known, but apparently it does not appear clearly in the literature.

Proposition 2.3. Let $\omega$ be a non empty open subset of $\Omega$ and $T>0$. Then the equation (1.1) is approximately controllable in $L^{2}(\Omega)$ in the sense that for any $y_{0} \in L^{2}(\Omega)$, the set of reachable states $\left\{y(T), v \in L^{2}\left(0, T ; L^{2}(\omega)\right)\right\}$ is dense in $L^{2}(\Omega)$. 
Proof. First of all, by translation, we can restrict ourselves to the case $y_{0}=0$. We introduce the (backward) adjoint equation

$$
\mid \begin{array}{ll}
i \frac{\partial \varphi}{\partial t}+\Delta \varphi=0 & \text { in } \Omega \times(0, T) \\
\varphi=0 & \text { on } \Gamma \times(0, T) \\
\varphi(T)=\varphi_{T} & \text { in } \Omega
\end{array}
$$

where $\varphi_{T} \in L^{2}(\Omega)$.

We notice that

$$
-i \frac{\partial \bar{\varphi}}{\partial t}+\Delta \bar{\varphi}=0 \text { in } \Omega \times(0, T)
$$

Multiplying (1.1) by $\varphi$ and integrating by parts we get

$$
i \int_{\Omega} y(T) \bar{\varphi}_{T} \mathrm{~d} x=\int_{(0, T) \times \omega} v \bar{\varphi} \mathrm{d} x \mathrm{~d} t .
$$

Let $\varphi_{T}$ be in the orthogonal in $L^{2}(\Omega)$ of $\left\{y(T), v \in L^{2}\left(0, T ; L^{2}(\omega)\right)\right\}$. Then we must have

$$
\forall v \in L^{2}\left(0, T ; L^{2}(\omega)\right), \int_{(0, T) \times \omega} v \bar{\varphi} \mathrm{d} x \mathrm{~d} t=0
$$

which implies

$$
\varphi=0 \text { in } \omega \times(0, T) .
$$

A unique continuation result has been obtained by Masuda [13] for the Schrödinger equation in the whole space, but his argument, which mainly concerns the time variable, may be adapted without major changes to the case of a bounded domain. The unique continuation property can also be found in Hörmander [6], Theorem 8.6.8. The unique continuation result implies

$$
\varphi=0 \text { in } \Omega \times(0, T)
$$

which in turn implies that $\varphi_{T}=0$. Therefore the orthogonal set of $\left\{y(T), v \in L^{2}\left(0, T ; L^{2}(\omega)\right)\right\}$ is reduced to $\{0\}$ and the proof of Proposition 2.3 is complete.

Remark 2.4. As a consequence of this result we obtain the fact, which was announced, that in (2.2), we can choose $v$ such that $\int_{D}|w(v)|^{2}(T, x) \mathrm{d} x \neq 0$.

Now Proposition 2.3 immediately implies Theorem 2.1 because, by density, given $\alpha>0$, we can find $v \in$ $L^{2}\left(0, T ; L^{2}(\omega)\right)$ such that

$$
|y(T)|_{L^{2}(\Omega)}^{2} \leq \alpha
$$

which implies of course

$$
|y(T)|_{L^{2}(D)}^{2} \leq \alpha .
$$


We just have to prove that this can be achieved with a control $v$ such that $\int_{D}|w(v)|^{2}(T, x) \mathrm{d} x \neq 0$. Suppose this is not the case. Then we can repeat the argument for any $\alpha^{\prime}$ with $0<\alpha^{\prime} \leq \alpha$ and this implies that

$$
\int_{D}|z(T, x)|^{2} \mathrm{~d} x=0
$$

Then we obtain for any $v$ such that $\int_{D}|w(v)|^{2}(T, x) \mathrm{d} x \neq 0$,

$$
\int_{D}|y(\lambda v)(T, x)|^{2} \mathrm{~d} x=\int_{D}\left|y_{\lambda}(v)(T, x)\right|^{2} \mathrm{~d} x=|\lambda|^{2} \int_{D}|w(v)(T, x)|^{2} \mathrm{~d} x .
$$

This shows that we can achieve our result by choosing only $|\lambda|$.

\section{CASE OF the WAVE EQUATion}

Throughout this section the functions have real values.

\subsection{Main result and relation between localized energy and localized controllability}

We suppose here that $\Omega$ is $C^{\infty}$ and we introduce the generalized bicharacteristics for the wave operator as defined in [8], We assume that these bicharacteristics have no contact of infinite order with the boundary of $\Omega \times(0, T)$ and from their definition, they are continuous with respect to their initial datas.

We introduce here a localized geometric control condition (LGCC) on $D, \omega$ and $T_{0}>0$ as follows.

Condition 3.1 (LGCC). Every generalized bicharacteristics associated with the wave operator starting at the point $\left(\left(0, x_{0}\right),\left(\tau_{0}, \xi_{0}\right)\right)$ with $x_{0} \in \bar{D}$ does not hit the boundary of $(0, T) \times \Omega$ at a diffractive point and meets $\left(0, T_{0}\right) \times \omega \times \mathbb{R}^{N+1}$.

Another way of writing this condition is to say that every ray of the geometric optics starting from a point $x_{0} \in \bar{D}$ at time $t=0$ does not hit the boundary of $\Omega$ at a diffractive point and meets $\omega$ before time $T_{0}$.

We can notice that this condition is written in a slightly different way as Condition 1.1 in [5] and this will be important in the proof of the theorem stated below.

Our main result concerning the wave equation is the following.

Theorem 3.2. Let $T>T_{0}$ and let us assume that $D, \omega$ and $T_{0}$ satisfy the localized geometric condition ( $\left.L G C C\right)$. Then, for any $y_{0} \in H_{0}^{1}(\Omega)$ and $y_{1} \in L^{2}(\Omega)$, there exists a control $u \in L^{2}\left(0, T ; L^{2}(\omega)\right)$ such that for any $\alpha \geq 0$, there exists $\lambda \in \mathbb{R}$ such that by choosing $v=\lambda u$, the solution $y$ of the controlled wave equation (1.4) satisfies

$$
E_{D}^{W}(y)(T)=\alpha
$$

A particular case is $\alpha=0$, case of exact localized controllability, which implies that there exists a control $v \in L^{2}\left(0, T ; L^{2}(\omega)\right)$ such that

$$
y(T)=0 \text { and } \frac{\partial y}{\partial t}(T)=0 \text { in } D .
$$

In a first step we will follow the lines of the previous case of the Schrödinger equation. Let us split the solution $y$ of (1.4) in two parts

$$
y=y(v)=z+w(v),
$$


with $z$ and $w(v)$ solutions of

$$
\mid \begin{array}{ll}
\frac{\partial^{2} z}{\partial t^{2}}-\Delta z=0 & \text { in } \Omega \times(0, T) \\
z=0 & \text { on } \Gamma \times(0, T) \\
z(0)=y_{0} ; & \frac{\partial z}{\partial t}(0)=y_{1}
\end{array},
$$

and, respectively,

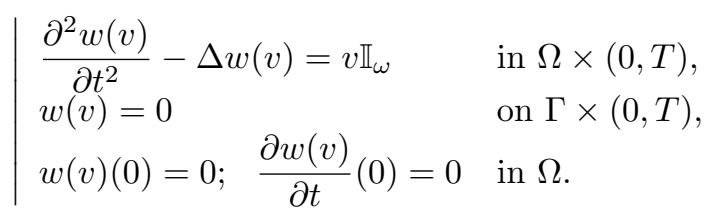

We know that

$$
E_{D}^{W}(z)(T) \leq E_{\Omega}^{W}(z)(T)=E_{\Omega}^{W}(z)(0)=\frac{1}{2} \int_{\Omega}\left(\left|y_{1}(x)\right|^{2}+\left|\nabla y_{0}(x)\right|^{2}\right) \mathrm{d} x
$$

and

$$
\forall \lambda \in \mathbb{R}, w(\lambda v)=\lambda w(v)
$$

Define

$$
y_{\lambda}(v)=y(\lambda v)=z+\lambda w(v) .
$$

We have

$$
\begin{aligned}
E_{D}^{W}\left(y_{\lambda}(v)\right)(T)= & E_{D}^{W}(z)(T) \\
& +\lambda\left(\left(\frac{\partial z}{\partial t}(T), \frac{\partial w(v)}{\partial t}(T)\right)_{L^{2}(D)}+(\nabla z(T), \nabla w(v)(T))_{L^{2}(D)}\right) \\
& +\lambda^{2} E_{D}^{W}(w(v))(T) .
\end{aligned}
$$

We will see later on (see Rem. 3.4) that we can choose $u$ such that $E_{D}^{W}(w(u))(T) \neq 0$.

First case $E_{D}^{W}(z)(T)=0$.

In this case, we have immediately

$$
E_{D}^{W}\left(y_{\lambda}(u)\right)(T)=\lambda^{2} E_{D}^{W}(w(u))(T)
$$

and this shows that by choosing $\lambda$ we can obtain

$$
E_{D}^{W}\left(y_{\lambda}(u)\right)(T)=\alpha .
$$

Second case $E_{D}^{W}(z)(T) \neq 0$.

We see that

$$
E_{D}^{W}\left(y_{\lambda}(u)\right)(T) \rightarrow+\infty \text { when } \lambda \rightarrow+\infty .
$$


We will show in the next section that we can find $u$ such that

$$
y(u)(T)=0 \text { and } \frac{\partial y(u)}{\partial t}(T)=0 \text { in } D,
$$

which we call exact localized controllability. This will imply

$$
E_{D}^{W}(w(u))(T)=E_{D}^{W}(z)(T) \neq 0
$$

Therefore, for any $\alpha \geq 0$, by choosing $\lambda \in \mathbb{R}$ we find $v=\lambda u$ such that $E_{D}^{W}(y(v))(T)=\alpha$.

\subsection{Exact localized controllability for the wave equation}

We are going to prove the following localized controllability result which is in fact the last part of Theorem 3.2.

Theorem 3.3. We assume that $D, \omega$ and $T_{0}$ satisfy condition (LGCC) and we take $T>T_{0}$. Then for any $y_{0} \in H_{0}^{1}(\Omega)$ and $y_{1} \in L^{2}(\Omega)$, there exists a control $u \in L^{2}\left(0, T: L^{2}(\omega)\right)$ such that the solution $y(u)$ of equation (1.4) with control $u$ satisfies

$$
y(u)(T)=0 \quad \text { and } \quad \frac{\partial y(u)}{\partial t}(T)=0 \text { in } D .
$$

Remark 3.4. The result of this Theorem 3.3 implies that $E_{D}^{W}(w(u))(T)=E_{D}^{W}(z)(T)$. In addition, by solving backward equation (3.3) for $z$ with (final) data supported in $D$ with non zero energy, we can find initial data $y_{0}$ and $y_{1}$ such that $E_{D}^{W}(z)(T) \neq 0$. Applying now Theorem 3.3 to these initial data, we obtain a control $u$ such that $E_{D}^{W}(w(u))(T) \neq 0$ as announced earlier.

We adapt here Lions' Hilbert Uniqueness Method (HUM) (see [11]) and we introduce the adjoint (backward) equation for initial values having their support in $D$. Let us take

$$
\varphi_{0} \in H_{0}^{1}(D) \text { and } \varphi_{1} \in L^{2}(D)
$$

which are extended by 0 (with the same notation) in $\Omega \backslash D$ and let $\varphi$ be solution of

$$
\mid \begin{array}{ll}
\frac{\partial^{2} \varphi}{\partial t^{2}}-\Delta \varphi=0 & \text { in } \Omega \times(0, T), \\
\varphi=0 & \text { on } \Gamma \times(0, T) \\
\varphi(T)=\varphi_{0}, \frac{\partial \varphi}{\partial t}(T)=\varphi_{1} & \text { in } \Omega .
\end{array}
$$

We know that

$$
\varphi \in C\left([0, T] ; H_{0}^{1}(\Omega)\right) \cap C^{1}\left([0, T] ; L^{2}(\Omega)\right)
$$

and

$$
E_{\Omega}^{W}(\varphi)(0)=E_{\Omega}^{W}(\varphi)(T)=E_{D}^{W}(\varphi)(T)=\frac{1}{2} \int_{D}\left(\left|\varphi_{1}(x)\right|^{2}+\left|\nabla \varphi_{0}(x)\right|^{2}\right) \mathrm{d} x
$$

We are going to prove the following observability inequality. 
Theorem 3.5. We assume that $D, \omega$ and $T_{0}$ satisfy condition (LGCC) and we take $T>T_{0}$. Then there exists a constant $C>0$ such that for every $\varphi_{0} \in H_{0}^{1}(D)$ and $\varphi_{1} \in L^{2}(D)$, we have

$$
E_{\Omega}^{W}(\varphi)(0)=E_{D}^{W}(\varphi)(T) \leq C \int_{(0, T) \times \omega}\left|\frac{\partial \varphi}{\partial t}(t, x)\right|^{2} \mathrm{~d} x \mathrm{~d} t .
$$

Let us assume for the moment that Theorem 3.5 is proved. It is well known that we also have the converse inequality [11]

$$
\int_{(0, T) \times \omega}\left|\frac{\partial \varphi}{\partial t}(t, x)\right|^{2} \mathrm{~d} x \mathrm{~d} t \leq C^{\prime} E_{\Omega}^{W}(\varphi)(0)
$$

We can apply Lax-Milgram Theorem to the bilinear form

$$
\int_{(0, T) \times \omega} \frac{\partial \hat{\varphi}}{\partial t}(t, x) \frac{\partial \varphi}{\partial t}(t, x) \mathrm{d} x \mathrm{~d} t
$$

Then, for every $y_{0} \in H_{0}^{1}(\Omega)$ and $y_{1} \in L^{2}(\Omega)$, there exists $\tilde{\varphi}_{0} \in H_{0}^{1}(D)$ and $\tilde{\varphi}_{1} \in L^{2}(D)$ such that denoting by $\tilde{\varphi}$ the solution of (3.6) corresponding to these initial values, we have for every $\varphi_{0} \in H_{0}^{1}(D)$ and $\varphi_{1} \in L^{2}(D)$,

$$
\left(\left(y_{0}, \varphi(0)\right)_{H_{0}^{1}(\Omega)}-\left(y_{1}, \frac{\partial \varphi}{\partial t}(0)\right)_{L^{2}(\Omega)}=\int_{(0, T) \times \omega} \frac{\partial \tilde{\varphi}}{\partial t}(t, x) \frac{\partial \varphi}{\partial t}(t, x) \mathrm{d} x \mathrm{~d} t .\right.
$$

Taking the control $u=\frac{\partial \tilde{\varphi}}{\partial t}$ in (1.4) and multiplying this equation by $\frac{\partial \varphi}{\partial t}$ (this looks formal but can be easily and classically justified) we obtain

$$
\begin{aligned}
& \left(\frac{\partial y}{\partial t}(T), \varphi_{1}\right)_{L^{2}(\Omega)}-\left(y_{1}, \frac{\partial \varphi}{\partial t}(0)\right)_{L^{2}(\Omega)}-\left(\left(y(T), \varphi_{0}\right)\right)_{H_{0}^{1}(\Omega)}+\left(\left(y_{0}, \varphi(0)\right)_{H_{0}^{1}(\Omega)}\right. \\
& =\int_{(0, T) \times \omega} \frac{\partial \tilde{\varphi}}{\partial t}(t, x) \frac{\partial \varphi}{\partial t}(t, x) \mathrm{d} x \mathrm{~d} t .
\end{aligned}
$$

This implies that for every $\varphi_{0} \in H_{0}^{1}(D)$ and $\varphi_{1} \in L^{2}(D)$,

$$
\left(\frac{\partial y}{\partial t}(T), \varphi_{1}\right)_{L^{2}(\Omega)}-\left(\left(y(T), \varphi_{0}\right)\right)_{H_{0}^{1}(\Omega)}=0
$$

which in turn implies

$$
y(T)=0 \text { and } \frac{\partial y}{\partial t}(T)=0 \text { in } D
$$

and finishes the proof of Theorem 3.3.

An immediate consequence of Theorem 3.3 is the following.

Corollary 3.6. We assume the same hypotheses as in Theorem 3.3. Let $z_{0} \in H_{0}^{1}(\Omega)$ and $z_{1} \in L^{2}(\Omega)$. Then there exists a control $\tilde{u} \in L^{2}\left(0, T ; L^{2}(\omega)\right)$ such that the solution $y(\tilde{u})$ of equation (1.4) with control $\tilde{u}$ satisfies

$$
y(\tilde{u})(T)=z_{0} \text { and } \frac{\partial y(\tilde{u})}{\partial t}(T)=z_{1} \text { in } D .
$$


Proof of Corollary 3.6. Let $\tilde{z}$ be solution of the backward problem

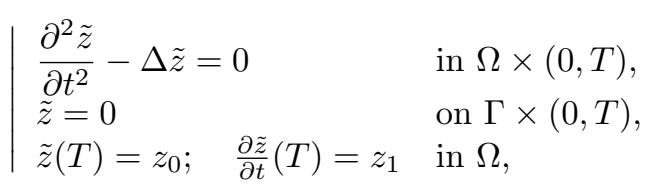

Then $\tilde{z} \in C\left([0, T] ; H_{0}^{1}(\Omega)\right) \cap C^{1}\left([0, T] ; L^{2}(\Omega)\right)$. Let us define

$$
\tilde{y}_{0}=y_{0}-\tilde{z}(0) \text { and } \tilde{y}_{1}=y_{1}-\frac{\partial \tilde{z}}{\partial t}(0),
$$

and apply Theorem 3.3 to the initial data $\tilde{y}_{0}$ and $\tilde{y}_{1}$. We obtain a control $\tilde{u} \in L^{2}\left(0, T ; L^{2}(\omega)\right)$ such that the corresponding solution $\tilde{y}$ of (1.4) satisfies

$$
\tilde{y}(T)=0, \text { and } \frac{\partial \tilde{y}}{\partial t}(T)=0
$$

Now

$$
y(\tilde{u})=\tilde{y}+\tilde{z}
$$

and the control $\tilde{u}$ satisfy the conclusion of Corollary 3.6.

We still have to prove Theorem 3.5.

Proof of Theorem 3.5. Our strategy of proof is classical and follows the lines of the various works for (global) exact controllability of the wave equation by [8] or [3], see also [1]. In order to simplify the presentation we change the variable $t$ in $T-t$ in order to consider the forward problem instead of the backward problem. We also write

$$
E_{D}^{W}\left(\varphi_{0}, \varphi_{1}\right)=E_{D}^{W}(\varphi)(0)
$$

Let us first prove a weak observability inequality, namely

$$
\begin{aligned}
& \exists C>0, \forall\left(\varphi_{0}, \varphi_{1}\right) \in H_{0}^{1}(D) \times L^{2}(D) \\
& E_{D}^{W}\left(\varphi_{0}, \varphi_{1}\right) \leq C \int_{(0, T) \times \omega}\left|\frac{\partial \varphi}{\partial t}(t, x)\right|^{2} \mathrm{~d} x \mathrm{~d} t+C\left\|\left(\varphi_{0}, \varphi_{1}\right)\right\|_{L^{2}(\Omega) \times H^{-1}(\Omega)}^{2}
\end{aligned}
$$

We argue by contradiction. Suppose (3.10) is not true. Then there exists a sequence $\left(\varphi_{0}^{n}, \varphi_{1}^{n}\right) \in H_{0}^{1}(D) \times L^{2}(D)$ such that

$$
E_{D}^{W}\left(\varphi_{0}^{n}, \varphi_{1}^{n}\right)=1
$$

and

$$
\int_{(0, T) \times \omega}\left|\frac{\partial \varphi^{n}}{\partial t}(t, x)\right|^{2} \mathrm{~d} x \mathrm{~d} t+\left\|\left(\varphi_{0}^{n}, \varphi_{1}^{n}\right)\right\|_{L^{2}(\Omega) \times H^{-1}(\Omega)}^{2} \rightarrow 0
$$


After an extraction of a subsequence, still denoted in the same way, we know that

$$
\begin{gathered}
\int_{(0, T) \times \omega}\left|\frac{\partial \varphi^{n}}{\partial t}(t, x)\right|^{2} \mathrm{~d} x \mathrm{~d} t \rightarrow 0, \\
\left(\varphi_{0}^{n}, \varphi_{1}^{n}\right) \rightarrow\left(\varphi_{0}, \varphi_{1}\right) \text { in } H_{0}^{1}(\Omega) \times L^{2}(\Omega) \text { weakly, } \\
\left(\varphi_{0}^{n}, \varphi_{1}^{n}\right) \rightarrow\left(\varphi_{0}, \varphi_{1}\right) \text { in } L^{2}(\Omega) \times H^{-1}(\Omega) \text { strongly },
\end{gathered}
$$

and

$$
\left\|\left(\varphi_{0}^{n}, \varphi_{1}^{n}\right)\right\|_{L^{2}(\Omega) \times H^{-1}(\Omega)}^{2} \rightarrow 0 .
$$

Therefore

$$
\left(\varphi_{0}, \varphi_{1}\right)=0
$$

We can also easily show that for every $\theta \in C_{0}^{\infty}((0, T) \times \omega)$

$$
\int_{(0, T) \times \omega} \theta\left(\left|\frac{\partial \varphi^{n}}{\partial t}\right|^{2}+\left|\nabla \varphi^{n}\right|^{2}\right) \mathrm{d} x \mathrm{~d} t \rightarrow 0 .
$$

We identify the initial values of the wave equation to the corresponding solutions and we now use the notations of [8] (see also $[9,10]$ ). Let $Y=\bar{\Omega} \times(0, T)$ and ${ }^{b} T Y$ be the fiber bundle of rank $N+1$ whose sections are the vector fields tangent to $\partial Y$. Let ${ }^{b} T^{*} Y$ be its dual fiber bundle. We denote by $j$ the natural projection of $T^{*} Y$ on ${ }^{b} T^{*} Y$ and by $\Sigma$ the image by $j$ of the characteristic set of the wave equation. We set $\hat{\Sigma}=\Sigma \cup j\left(T^{*} Y_{/ \partial Y}\right)$ and $S^{*} \hat{\Sigma}=\hat{\Sigma} / \mathbb{R}_{+}^{*}$ (the quotient applies only to the frequency variables). For a subsequence again, still denoted in the same way, there exists a microlocal defect measure $\mu$ defined on $S^{*} \hat{\Sigma}$ associated to the sequence $\varphi^{n}$. From (3.11), we know that $\mu$ vanishes on $\omega \times(0, T) \times \mathbb{R}^{N+1}$. On the other hand, we know from [8] that $\mu$ is invariant by the flow of the generalized bicharacteristics.

Let us show that all the bicharacteristics emanating from a point outside $\bar{D}$ at $t=0$ are not contained in $\operatorname{Supp}(\mu)$. For $x_{0} \notin \bar{D}$ the initial conditions vanish in a neighborhood of $x_{0}$ and therefore, due to the finite speed propagation for the wave equation, for $t$ small enough, the solutions vanish in a neighborhood of $\left(t, x_{0}\right)$ which shows that for any $\left(\tau_{0}, \xi_{0}\right) \in \mathbb{R}^{N+1}, \mu$ vanishes on $\left(\left(t, x_{0}\right),\left(\tau_{0}, \xi_{0}\right)\right)$. Now for $(t, x) \in(0, T) \times \Omega$ and for $(\tau, \xi) \in \mathbb{R}^{N+1}$ with $((t, x),(\tau, \xi)) \in \operatorname{Supp}(\mu)$, the whole bicharacteristics coming to this point belongs to $\operatorname{Supp}(\mu)$, in particular for $t=0,\left(\left(0, x_{0}\right),\left(\tau_{0}, \xi_{0}\right)\right) \in \operatorname{Supp}(\mu)$ where $\left(\tau_{0}, \xi_{0}\right)$ are the corresponding initial conditions for this bicharacteristics. As seen before, this implies that $x_{0} \in \bar{D}$

On the other hand, from condition (LGCC), for every $x_{0} \in \bar{D}$, the bicharacteristics emanating from $\left(0, x_{0}\right)$ meet $(0, T) \times \omega$, which implies that $\left(\left(0, x_{0}\right),(\tau, \xi)\right) \notin \operatorname{Supp}(\mu)$. Therefore $\mu=0$ and we must have

$$
E_{D}^{W}\left(\varphi_{0}^{n}, \varphi_{1}^{n}\right) \rightarrow 0
$$

which gives a contradiction. This proves the weak observability inequality (3.10).

We still need to prove the (strong) observability inequality (3.7).

We can rewrite inequality (3.10) in the following way.

$$
\exists C>0, \forall\left(\varphi_{0}, \varphi_{1}\right) \in H_{0}^{1}(D) \times L^{2}(D),
$$




$$
\int_{(0, T) \times \omega}\left|\frac{\partial \varphi}{\partial t}(t, x)\right|^{2} \mathrm{~d} x \mathrm{~d} t \geq C E_{D}^{W}\left(\varphi_{0}, \varphi_{1}\right)-\left\|\left(\varphi_{0}, \varphi_{1}\right)\right\|_{L^{2}(\Omega) \times H^{-1}(\Omega)}^{2}
$$

On the other hand, $E_{D}^{W}\left(\varphi_{0}, \varphi_{1}\right)$ is the square of a norm in $H_{0}^{1}(\Omega) \times L^{2}(\Omega)$ for functions having their support in $D$ and because $\Omega$ is bounded, the embedding $H_{0}^{1}(\Omega) \times L^{2}(\Omega) \subset L^{2}(\Omega) \times H^{-1}(\Omega)$ is compact. Then by a standard compactness-uniqueness argument, (3.12) will imply (3.7) if the kernel

$$
N=\left\{\left(\varphi_{0}, \varphi_{1}\right) \in H_{0}^{1}(D) \times L^{2}(D), \frac{\partial \varphi}{\partial t}(t, x)=0 \text { in }(0, T) \times \omega\right\}
$$

is reduced to $\{0\}$. This constitutes the major point of the proof of Theorem 3.5.

Let $\left(\varphi_{0}, \varphi_{1}\right) \in N$. Then from (3.12) there exists $C>0$ independent of $\left(\varphi_{0}, \varphi_{1}\right)$ such that

$$
E_{D}^{W}\left(\varphi_{0}, \varphi_{1}\right) \leq C||\left(\varphi_{0}, \varphi_{1}\right) \|_{L^{2}(\Omega) \times H^{-1}(\Omega)}^{2} .
$$

Because of the compact embedding this implies that $N$ is a finite dimensional vector space. We are going to prove that the operator $\Delta$ operates in $N$. We first show that $\left(\varphi_{1}, \Delta \varphi_{0}\right) \in N$.

For $0<h<h_{0}$ with $h_{0}$ small, we consider the differential quotient

$$
\varphi_{h}(t, x)=\frac{\varphi(t+h, x)-\varphi(t, x)}{h}
$$

and

$$
\begin{gathered}
\varphi_{h, 0}(x)=\frac{\varphi(h, x)-\varphi_{0}(x)}{h}, \\
\varphi_{h, 1}(x)=\frac{\frac{\partial \varphi}{\partial t}(h, x)-\varphi_{1}(x)}{h} .
\end{gathered}
$$

Then $\varphi_{h}$ is solution of

$$
\begin{aligned}
& \frac{\partial^{2} \varphi_{h}}{\partial t^{2}}-\Delta \varphi_{h}=0 \text { in }(0, T-h) \times \Omega, \\
& \varphi_{h}=0 \text { on }(0, T-h) \times \Gamma, \\
& \varphi_{h}(0)=\varphi_{h, 0}, \frac{\partial \varphi_{h}}{\partial t}(0)=\varphi_{h, 1} \text { in } \Omega .
\end{aligned}
$$

On the other hand we have

$$
\frac{\partial \varphi_{h}}{\partial t}(t, x)=0 \text { in }(0, T-h) \times \omega
$$

An important point is that in general $\operatorname{Supp}\left(\varphi_{h, 0}, \varphi_{h, 1}\right)$ is no longer contained in $D$. But for $h_{0}$ small enough and $0<h<h_{0}$, due to the finite speed propagation in the wave equation, it is contained in $D_{h}$ with

$$
D_{h} \subset \bigcup_{x \in D} B(x, \epsilon(h))
$$

where $\epsilon(h) \rightarrow 0$ when $h \rightarrow 0$. 
Therefore, using the continuity of the bicharacteristics with respect to their initial datas and the compactness of $\bar{D}$, we can assume that $h_{0}$ is small enough so that $D_{h}, \omega$ and $(T-h)$ still satisfy condition (LGCC). Repeating the previous argument, we obtain the existence of a constant $C_{1}>0$ such that for every $h, 0<h<h_{0}$,

$$
E_{D_{h}}^{W}\left(\varphi_{h, 0}, \varphi_{h, 1}\right) \leq C_{1}\left\|\left(\varphi_{h, 0}, \varphi_{h, 1}\right)\right\|_{L^{2}(\Omega) \times H^{-1}(\Omega)}^{2}
$$

Now we know that when $h \rightarrow 0$,

$$
\varphi_{h, 0} \rightarrow \frac{\partial \varphi}{\partial t}(0)=\varphi_{1} \text { in } L^{2}(\Omega)
$$

and

$$
\varphi_{h, 1} \rightarrow \frac{\partial^{2} \varphi}{\partial t^{2}}(0)=\Delta \varphi_{0} \text { in } H^{-1}(\Omega)
$$

This implies that $E_{D_{h}}^{W}\left(\varphi_{h, 0}, \varphi_{h, 1}\right)$ stays bounded when $h \rightarrow 0$ and shows that

$$
\varphi_{1} \in H_{0}^{1}(D), \text { and } \Delta \varphi_{0} \in L^{2}(D)
$$

If we define $\psi=\frac{\partial \varphi}{\partial t}$ we have

$$
\begin{aligned}
& \frac{\partial^{2} \psi}{\partial t^{2}}-\Delta \psi=0 \text { in }(0, T) \times \Omega, \\
& \psi=0 \text { on }(0, T) \times \Gamma \\
& \psi(0)=\varphi_{1}, \frac{\partial \psi}{\partial t}(0)=\Delta \varphi_{0} \text { in } \Omega
\end{aligned}
$$

and

$$
\frac{\partial \psi}{\partial t}(t, x)=0 \text { in }(0, T) \times \omega
$$

Therefore $\left(\varphi_{1}, \Delta \varphi_{0}\right) \in N$.

We can repeat the same argument to show that

$$
\left(\Delta \varphi_{0}, \Delta \varphi_{1}\right) \in N
$$

which shows that $\Delta$ operates from the finite dimensional vector space $N$ into $N$.

If $N$ is not reduced to $\{0\}$, the operator $\Delta$ has eigenfunctions $\left(u_{0}, u_{1}\right)$ associated to an eigenvalue $(-\lambda)$ with $\left(u_{0}, u_{1}\right) \in N$ and of course $u_{i}, i=0,1$ is an eigenfunction of $\Delta$ in $\Omega$ with Dirichlet boundary conditions. If $D$ is different from $\Omega$ this leads to a contradiction as $u_{i}$ has to vanish in $\Omega \backslash D$. Even if $D=\Omega$, we can write explicitely the solution $\varphi$ as

$$
\varphi(t, x)=\cos (\sqrt{\lambda} t) u_{0}(x)+\frac{1}{\sqrt{\lambda}} \sin (\sqrt{\lambda} t) u_{1}(x)
$$


Using the condition

$$
\frac{\partial \varphi}{\partial t}(t, x)=0 \text { in }(0, T) \times \omega
$$

we see that necessarily

$$
u_{0}(x)=0 \text { and } u_{1}(x)=0 \text { in } \omega,
$$

which implies

$$
u_{0}(x)=0 \text { and } u_{1}(x)=0 \text { in } \Omega
$$

and leads to a contradiction.

This proves that $N$ is reduced to $\{0\}$ and finishes the proof of Theorem 3.5 and therefore also the proofs of Theorem 3.3 and of Theorem 3.2.

Remark 3.7. The above argument cannot be applied to the case of Schrödinger equation for at least two reasons. First of all, we should make use of the semiclassical defect measure instead of the microlocal defect measure and this should be done on a small interval of time. But then, the argument cannot be continued as the propagation at infinite speed tells us that after a small interval of time the solution has a support on the whole domain $\Omega$ and the situation is no longer the same, neither analogous.

Acknowledgements. The authors want to thank Nicolas Burq for very helpful discussions and comments during the redaction of the present article. The authors want to thank the anonymous referees for their valuable remarks and corrections on the first version of this article. This research was initiated during the visit of the third author at the Universidade Federal de Pernambuco, Recife, supported by the Réseau Franco-Brésilien de Mathématiques.

\section{REFERENCES}

[1] C. Bardos, G. Lebeau and J. Rauch, Sharp sufficient conditions for the observation, control and stabilisation of waves from the boundary. SIAM J. Control Optim. 305 (1992) 1024-1065.

[2] N. Burq, Contrôle de l'équation des ondes dans des ouverts peu réguliers. Asymptotic Anal. 14 (1997) $157-191$.

[3] N. Burq and P. Gerard, Condition nécessaire et suffisante pour la contrôlabilité exacte des ondes. C.R. Acad. Sci. 325 (1997) 749-752.

[4] P. Gerard, Microlocal defect measures. Commun. Partial Differ. Equ. 16 (1991) 1761-1794.

[5] Y. He, Partial exact controllability for wave equations. Syst. Control Lett. 103 (2017) 45-49.

[6] L. Hörmander, The Analysis of Linear Partial Differential Operators. I. Distribution Theory and Fourier Analysis. Classics Math. Reprint of the second (1990) edition. Springer-Verlag, Berlin (2003).

[7] G. Lebeau, Contrôle de l'equation de Schrödinger. J. Math. Pures Appl. 71 (1992) 267-291.

[8] G. Lebeau, Equation des ondes amorties. Algebraic and Geometric Methods in Mathematical Physics, 73-109, Edited by A. Boutet de Monvel and V. Marchenko. Kluwer Academic, The Netherlands (1996).

[9] J. Le Rousseau, G. Lebeau, P. Terpolilli and E. Trelat, Geometric control condition for the wave equation with a time-dependent observation domain. Anal. Partial Differ. Equ. 10 (2017) 983-1015.

[10] C. Letrouit, Infinite-time observability of the wave equation with time-varying observation domains under a geodesic recurrence condition. Preprint hal-0210213 (2019).

[11] J.-L. Lions, Contrôlabilité exacte, perturbations et stabilisation des systèmes distribués. Tome 1, Contrôlabilité exacte. Collection R.M.A 8, Masson (1988).

[12] E. Machtyngier, Exact controllability for the Schrödinger equation. SIAM J. Control and Optim. 32 (1994) 24-34.

[13] K. Masuda, A Unique Continuation Theorem for Solutions of the Schrödinger Equations. Proc. Jpn. Acad. 125 (1967) 853-860. 